18,07

\title{
Влияние механического растяжения на адсорбционные свойства легированного азотом графена
}

\author{
(С) И.Ю. Долинский ${ }^{1}$, К.П. Катин ${ }^{1,2, \uparrow}$, К.С. Гришаков ${ }^{1,2}$, В.С. Прудковский ${ }^{2,3}$, \\ Н.И. Каргин ${ }^{1}$, М.М. Маслов ${ }^{1,2}$ \\ ${ }^{1}$ Национальный исследовательский ядерный университет „МИФИ“, \\ Москва, Россия \\ ${ }^{2}$ University of Crete, \\ Heraklion, Greece \\ ${ }^{3}$ Научно-исследовательский институт проблем развития \\ научно-образовательного потенциала молодежи, \\ Москва, Россия \\ ฯ E-mail: kpkatin@yandex.ru \\ (Поступила в Редакцию 5 июля 2017 г. \\ В окончательной редакции 15 октября 2017 г.)
}

\begin{abstract}
Представлены результаты квантово-химического моделирования хемосорбции атомарного водорода, а также эпоксидной, карбоксильной и гидроксильной функциональных групп на легированный азотом графен. Показано, что замещающий атом азота не связывается с адсорбирующимися группами непосредственно, но значительно увеличивает адсорбционную активность соседних атомов углерода. Механическое растяжение легированного графена приводит к понижению энергии адсорбции всех рассмотренных радикалов. Величина этого понижения для эпоксидной группы значительно выше, чем для остальных функциональных групп. Полученные результаты подтверждают, что при достаточном растяжении легированного азотом графенового листа реакция диссоциации молекулярных водорода и кислорода с последующим осаждением получившихся радикалов на графен может быть энергетически выгодной.
\end{abstract}

Работа поддержана РФФИ (проект № 16 32-60081 mol_a_dk).

DOI: $10.21883 /$ FTT.2018.04.45699.220

\section{1. Введение}

После открытия графена в 2004 году [1], его уникальные механические, термические, оптические и электронные свойства привлекают большой интерес исследователей [2-5]. Для эффективного использования графена в наноэлектронике и других прикладных областях необходимо научиться управляемо модифицировать его электронное строение, химическую активность и другие характеристики. Этого можно достичь, располагая графеновые листы определенным образом друг относительно друга [6,7] или вводя в них структурные дефекты $[8,9]$, a также допируя их различными функциональными группами $[10,11]$. Еще одним распространенным способом „настройки“ свойств графена является замещающее легирование, при котором некоторые атомы углерода заменяются атомами азота, бора, серы, алюминия или других элементов. Чаще всего в качестве замещающего элемента используется азот [12].

Впервые $N$-легированный графен был синтезирован путем химического парофазного осаждения (CVD) в 2009 году [13]. Позднее были предложены и другие способы его получения, например, дуговой разряд [14] или пиролиз графена, функционализированного азотосодержащими группами [15]. Было показано, что азот может встраиваться в решетку графена, связываясь с тремя соседними атомами углерода, или образовывать более сложные дефекты (пиридинового или пирролического типа), приводящие к формированию „отверстийс в графеновом листе [13]. Простое встраивание азота в графеновую решетку значительно выгоднее энергетически, энергия формирования такого дефекта составляет всего $0.32 \mathrm{eV}[16]$. Это объясняется тем, что атомы углерода и азота имеют почти одинаковый размер, и длины C-C- и $\mathrm{C}-\mathrm{N}$-связей отличаются незначительно. Недавние исследование показало, что замещающий атом азота вызывает значительно меньшее напряжение графеновой решетки, чем, например, атом бора [17].

В настоящее время $N$-легированный графен рассматривается как перспективный материал, полезный для многих приложений [12]. Благодаря большой удельной площади, он может использоваться как эффективный катализатор, не содержащий благородных металлов $[18,19]$. Из графена, легированного азотом и серой, предлагается изготавливать аноды литий-ионных аккумуляторов [20]. Суперконденсаторы, созданные с использованием $N$-легированного графена, демонстрируют высокую удельную емкость до 250 F/g [21].

Особый интерес представляют адсорбционные свойства этого материала. Атом азота, встроенный в углеродную решетку, увеличивает химическую активность соседних атомов [22-24]. Это позволяет использовать $N$-легированный графен в качестве газового адсорбента [23], а также для хранения и транспортировки водоро- 


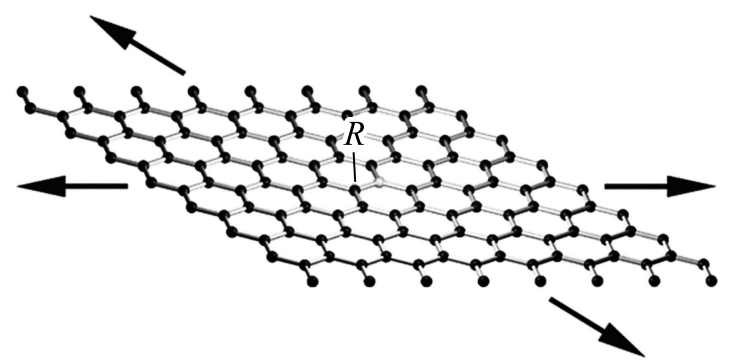

Рис. 1. Молекулярная структура сверхъячейки $N$-легированного графена $\mathrm{C}_{127} \mathrm{~N}$. Черные кружки означают атомы углерода, серый кружок - замещающий атом азота.

да [22]. Кроме того, в настоящее время разрабатывается электрохимический ДНК-биосенсор на основе $N$-легированного графена для поиска гена множественной лекарственной устойчивости человека, обнаружение которого приведет к прогрессу в лечении онкологических заболеваний $[25,26]$.

Настоящая работа посвящена исследованию адсорбции радикалов $\mathrm{H}, \mathrm{O}, \mathrm{OH}, \mathrm{COOH}$ на растянутый $N$-легированный графен. Перечисленные радикалы являются распространенными адсорбатами: Н интересен с точки зрения запасания водорода [27]; О и ОН играют важную роль при приготовлении, очистке и функционализации графена [28-31]; ОН и $\mathrm{COOH}$ обеспечивают его биосовместимость [32-34], СООН улучшает его адсорбционные свойства $[35,36]$. Механическое растяжение существенно меняет не только электронные [37], оптические [38] и термические $[39,40]$ свойства графена, но и его химическую активность [41]. Это позволяет управлять адсорбцией и миграцией радикалов по поверхности графена посредством механического воздействия [42,43]. В настоящей работе с помощью компьютерного моделирования рассматривается влияние внешнего растяжения на энергию связи $N$-легированного графена с адсорбентами.

\section{2. Методика расчета}

$N$-легированный графеновый лист моделировался при помощи ромбической $8 \times 8$ сверхъячейки $\mathrm{C}_{127} \mathrm{~N}$, содержащей 127 атомов углерода и один замещающий атом азота (рис. 1) с использованием периодических граничных условий. Такой размер ячейки достаточен для того, чтобы адсорбированный радикал и его образы, возникающие из-за периодических граничных условий, не взаимодействовали друг с другом [44]. Для описания межатомного взаимодействия использовалась неортогональная модель сильной связи $[45,46]$ с параметризацией [46]. Эта полуэмпирическая модель обладает высоким быстродействием по сравнению с $a b$ initio подходами, и корректно описывает графен, графан и их производные [47-50], а также фуллерены [51,52] и системы с углеродно-азотным каркасом $[53,54]$. Гео- метрическая оптимизация исходного и функционализированного $N$-легированного графена проводилась методом градиентного спуска: атомы смещались в направлении действующих на них сил, пока эти силы не становились меньше $0.001 \mathrm{eV} / \AA \AA$. Ограничения, связанные с симметрией системы, не принимались во внимание при проведении расчетов. Внешнее натяжение учитывалось посредством соразмерного (на одинаковое количество процентов) увеличения обоих периодов сверхъячейки. Энергия адсорбции $E_{\mathrm{ads}}$ для каждого радикала $R(R=\mathrm{H}, \mathrm{O}, \mathrm{OH}, \mathrm{COOH})$ вычислялась по формуле $E_{\text {ads }}=E\left(\mathrm{C}_{127} \mathrm{~N}+R\right)-E\left(\mathrm{C}_{127} \mathrm{~N}\right)-E(R)$, где $E\left(\mathrm{C}_{127} \mathrm{~N}+R\right), E\left(\mathrm{C}_{127} \mathrm{~N}\right)$ и $E(R)-$ энергии оптимизированных сверхъячейки с адсорбированным радикалом и изолированных сверхъячейки и радикала соответственно. Отрицательные значения $E_{\text {ads }}$ показывают, что в процессе адсорбции полная энергия системы понижается.

\section{3. Результаты и обсуждение}

Прежде всего мы рассмотрели адсорбцию радикалов на нерастянутый $N$-легированный графен. Наши расчеты подтвердили, что радикалы $\mathrm{H}, \mathrm{OH}$ и $\mathrm{COOH}$ связываются только с одним атомом адсорбента [55], а атомы О образует эпоксидную группу и располагаются над центром $\mathrm{C}-\mathrm{C}$ - или $\mathrm{C}-\mathrm{N}$-связи. Мы рассчитали энергии адсорбции каждого из радикалов $\mathrm{H}, \mathrm{OH}$ и $\mathrm{COOH}$ на семь различных узлов $N$-легированного графена, расположенных неэквивалентно относительно замещающего атома азота. Эти узлы отмечены как $A 0-A 6$ на рис. $2, a$, $A 0$ соответствует положению атома азота. В качестве места прикрепления эпоксидной группы рассматривались неэквивалентные пары узлов, отмеченные как $B 0-B 5$ на рис. $2, b$. Полученные энергии адсорбции представлены на рис. $3, a$ и $3, b$. Расчеты показывают, что радикалы $\mathrm{OH}$ и $\mathrm{H}$ способны прикрепляться непосредственно к атому азота, в отличие от группы СООН. Из рис. 3, $a$ видно, что наибольший выигрыш в энергии достигается при адсорбции на атом, являющийся ближайшим соседом
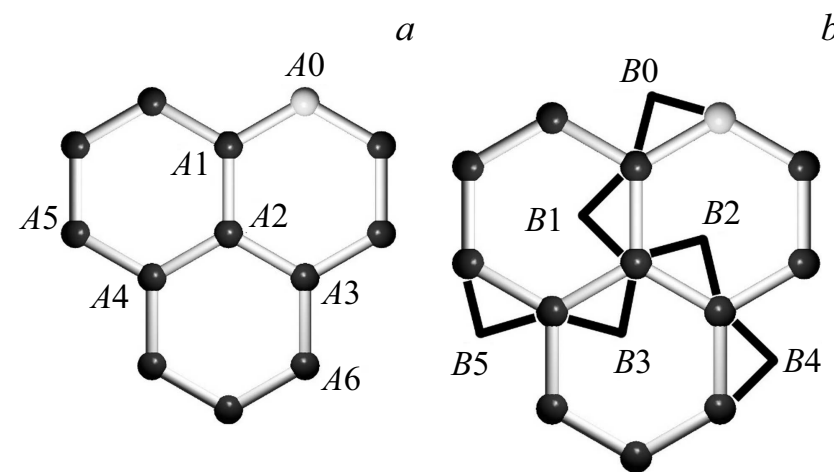

Рис. 2. Различные неэквивалентные расположения атомов $(a)$ и пар атомов $(b) N$-легированного графена вблизи замещающего атома азота. 

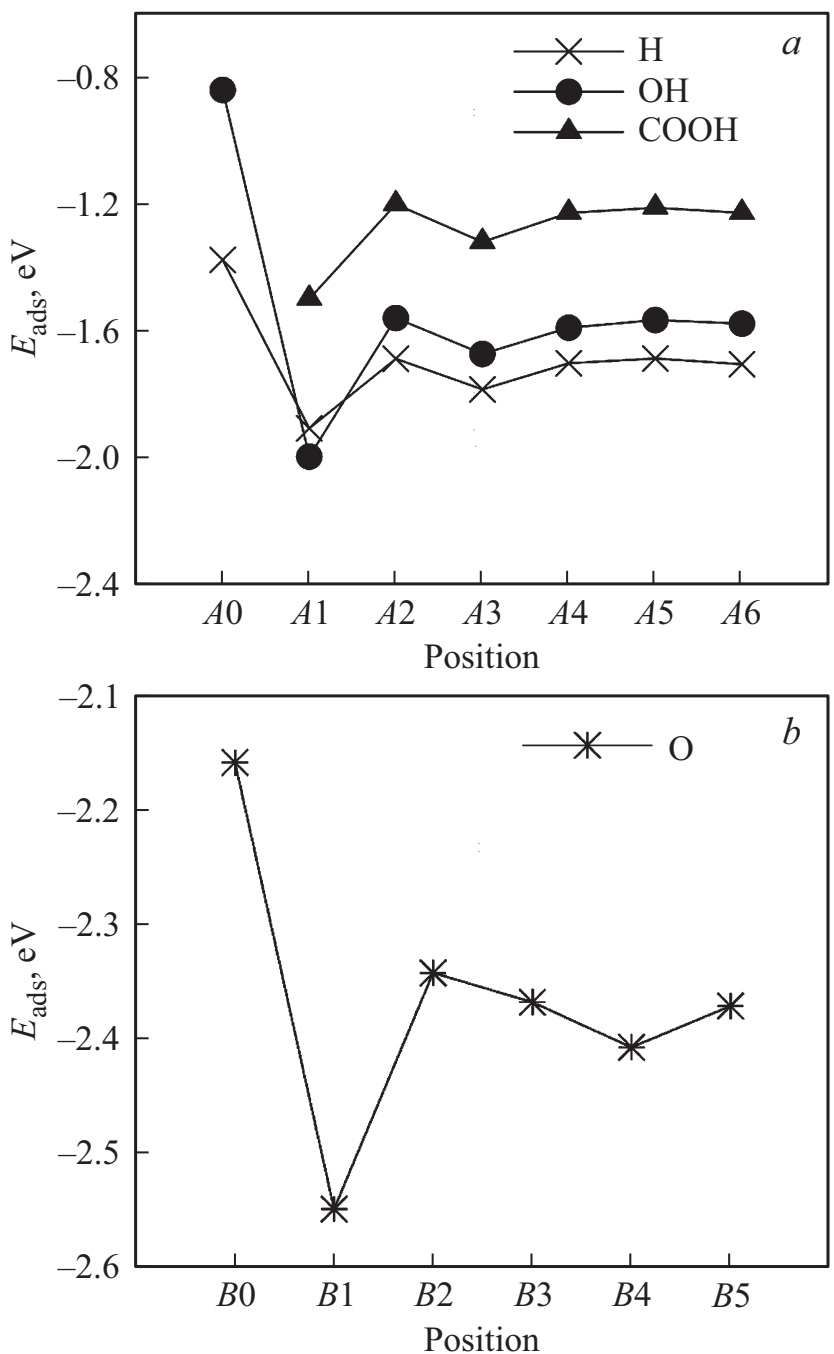

Рис. 3. Энергии адсорбции радикалов Н, ОН, СООН (a) и $\mathrm{O}(b)$ для их различных взаимных расположений на поверхности $N$-легированного графена относительно замещающего атома азота.

азота (отмеченный как $A 1$ на рис. $2, a)$. Этот результат подтверждается расчетом, недавно проведенным методом теории функционала плотности для адсорбированного атома водорода [17]. Отметим, что полученное в работе [17] значение энергии адсорбции водорода в положении $A 1$ равно $-1.9 \mathrm{eV}$, что хорошо совпадает с нашим результатом, представленном на рис. 3, $a$. Немонотонный характер графиков на рис. 3, $а$ позволяет обобщить правило минимального дисбаланса подрешеток, выполняющееся при одновременной адсорбции нескольких радикалов на графен [56,57], на случай $N$-легированного графена. В нашем случае его можно сформулировать следующим образом: больший энергетический выигрыш достигается в случае, когда адсорбция радикала и замещающий азотный дефект затрагивают различные графеновые подрешетки. Из графика на рис. $3, b$ видно, что для адсорбции атома кислорода наиболее выгодным также оказывается положение, ближайшее к атому азота и отмеченное как $B 1$ на рис. $2, b$.

Далее исследовалось влияние растяжения графена на энергию адсорбции радикалов $\mathrm{H}, \mathrm{OH}$ и $\mathrm{COOH}$ находящихся в положении $A 1$, а также радикала $\mathrm{O}$, находящегося в положении $B 1$. Другие положения радикалов значительно менее выгодны энергетически (см. рис. 3) и поэтому не рассматривались. Зависимость энергии адсорбции от растяжения графена представлена на рис. 4, $a$. Из этого рисунка видно, что при растяжении графена связь атома углерода с адсорбентом становится все более и более прочной. Изменения энергии адсорбции, вызванные растяжением, слабо различаются для радикалов $\mathrm{H}$, $\mathrm{OH}$ и $\mathrm{COOH}$ (см. рис. 4, a). Для эпоксидной группы О это изменение в $\sim 2$ раза больше, чем для остальных радикалов, что можно объяснить тем, что кислород образует с графеном две связи, а не одну. Отметим, что характер изменения энергии адсорбции, вызванный растяжением, слабо зависит от электроотрицательности и химических свойств адсорбируемого радикала.

При растяжении $N$-легированного графена с адсорбированными радикалами О и ОН на 5-7\% мы наблюдали необратимый разрыв $\mathrm{C}-\mathrm{N}$ связи, что приводило к возникновению дефектов, показанных на рис. $4, b, c$. Таким образом, C-N связь в $N$-легированном графене оказывается менее прочной, чем $\mathrm{C}-\mathrm{C}$ связи. Наши расчеты показывают, что наличие адсорбентов О или ОН вблизи
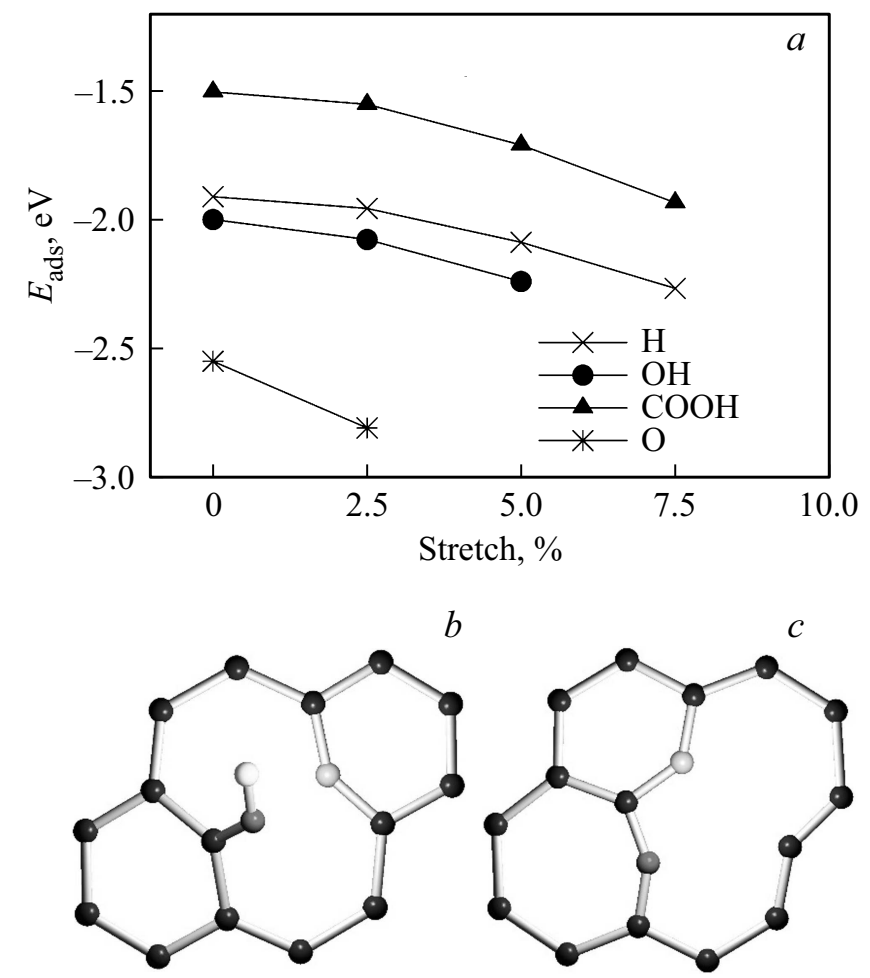

Рис. 4. Зависимость энергии адсорбции радикалов Н, ОН, $\mathrm{COOH}$ и О от величины обратимого растяжения $N$-легированного графена $(a)$. Необратимые дефекты, возникающие при растяжении $N$-легированного графена с адсорбированными радикалами $\mathrm{OH}(b)$ и $\mathrm{O}(c)$. 


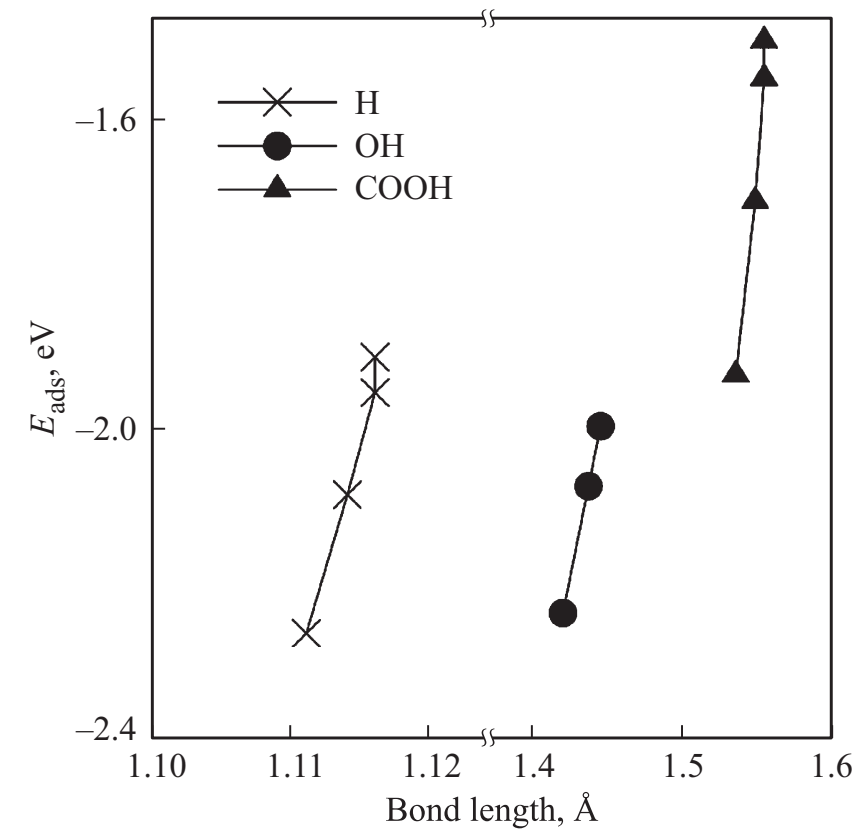

Рис. 5. Зависимость длин ковалентных связей $\mathrm{C}-\mathrm{H}, \mathrm{C}-\mathrm{O}$ и $\mathrm{C}-\mathrm{C}$ между атомом растянутого $N$-легированного графена и радикалами $\mathrm{H}, \mathrm{OH}$ и $\mathrm{COOH}$, соответственно, от энергии адсорбции. Разные точки графика, относящегося к одному и тому же радикалу, соответствуют разной степени растяжения графена.

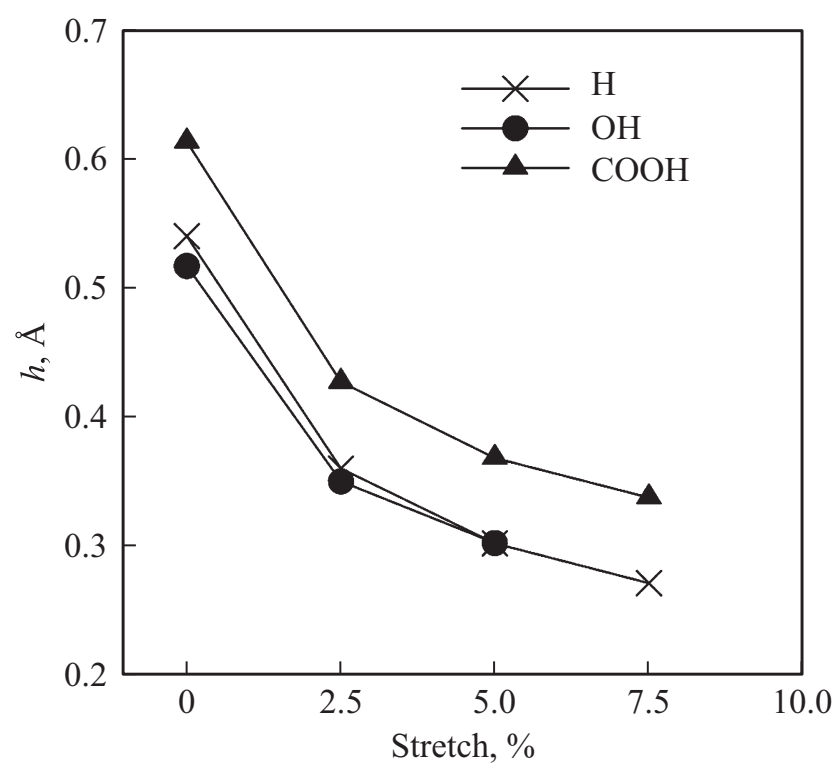

Рис. 6. Зависимость величины возвышения ковалентно связанного с радикалом Н, ОН или $\mathrm{COOH}$ атома углерода над плоскостью $N$-легированного графена от величины растяжения последнего.

внедренного атома азота дополнительно способствуют разрыву связи $\mathrm{C}-\mathrm{N}$.

Адсорбция радикала на графен сопровождается двумя процессами: образованием химической связи, приводя- щим к понижению полной энергии системы, и искривлением графенового листа, приводящим к ее повышению. Энергия адсорбции $E_{\text {ads }}$ определяется балансом между двумя указанными факторами. Растяжение графена влияет на оба процесса в сторону увеличения абсолютного значения $E_{\text {ads. }}$. Длина связи между адсорбентом и атомом графена монотонно уменьшается при растяжении, как показано на рис. 5, что подтверждает наличие химического вклада в изменение энергии адсорбции. С другой стороны, растяжение способствует „разглаживанию“ графена и возвращению его в недеформированное ,плоское“ состояние. На рис. 6 показано, что величина возвышения допированного атома углерода над плоскостью графенового листа монотонно убывает по мере растяжения для всех рассмотренных функциональных групп. Уменьшение энергии деформации, связанной со смещением атомов перпендикулярно плоскости графена, дополнительно повышает абсолютное значении энергии адсорбции.

\section{4. Заключение}

В настоящей работе мы исследовали адсорбцию радикалов на растянутый $N$-легированный графен. Расчеты показали, что растяжение способствует адсорбции, что характерно и для недопированного графена $[41,58]$. Однако поведение $N$-легированного графена демонстрирует несколько важных отличий. Во-первых, узлы $N$-легированного графена неэквивалентны, и адсорбция должна происходить преимущественно вблизи внедренного атома азота вне зависимости от химического состава и строения радикала. Во-вторых, $N$-легированный графен обладает меньшей прочностью и стремится к разрыву $\mathrm{C}-\mathrm{N}$-связей. Наличие радикалов способствует этому процессу, так что обратимое растяжение возможно только в пределах нескольких процентов (отметим, что „чистый“ графен выдерживает растяжение до 25\% [59]). Тем не менее, даже небольшого растяжения $\sim 5 \%$ достаточно для того, чтобы изменить энергию адсорбции на несколько десятых электрон-вольта. В-третьих, адсорбция на наиболее активные узлы $N$-легированного графена приводит к гораздо большему энергетическому выигрышу по сравнению с обычным графеном. Этот выигрыш сравним с энергией диссоциации устойчивых молекул $\mathrm{H}_{2}, \mathrm{O}_{2}, \mathrm{H}_{2} \mathrm{O}, \mathrm{HCOOH}$ и других. В зависимости от степени растяжения графена, диссоциация этих соединений с последующей адсорбцией на графен может оказаться как экзо-, так и эндотермической. Разумеется, для протекания таких реакций необходимо преодоление соответствующих энергетических барьеров, и их практическое осуществление, скорее всего, потребует специальных катализаторов. Тем не менее, в представленной работе продемонстрирована принципиальная возможность управления подобными реакциями посредством обратимого механического растяжения. 


\section{Список литературы}

[1] K.S. Novoselov, A.K. Geim, S.V. Morozov, D. Jiang, Y. Zhang, S.V. Dubonos, I.V. Grigorieva, A.A. Firsov. Science 306, 666 (2004).

[2] V. Chabot, D. Higgins, A. Yu, X. Xiao, Z. Chen, J. Zhang. Energy Environmental Sci. 7, 1564 (2014).

[3] G. Mittal, V. Dhand, K.Y. Rhee, S.J. Park, W.R. Lee. J. Industrial Eng. Chem. 21, 11 (2015).

[4] D. Higgins, P. Zamani, A. Yu, Z. Chen. Energy Environmental Sci. 9, 357 (2016).

[5] M.J. Allen, V.C. Tung, R.B. Kaner. Chem. Rev. 110, 132 (2010).

[6] A.V. Rozhkov, A.O. Sboychakov, A.L. Rakhmanov, F. Nori. Phys. Rev. B 95, 045119 (2017).

[7] Е.А. Беленков, В.А. Грешняков. ФТТ 57, 192 (2015).

[8] S.G. Rudi, R. Faez, M.K. Moravvej-Farshi. Superlattices Microstructures 100, 739 (2016).

[9] Е.А. Беленков, А.Е. Коченгин. ФТТ 57, 2071 (2015).

[10] C. Punckt, F. Muckel, S. Wolff, I.A. Aksay, C.A. Chavarin. Appl. Phys. Lett. 102, 023114 (2013).

[11] X. Tian, S. Sarkar, A. Pekker, M.L. Moser, I. Kalinina, E. Bekyarova, M.E. Itkis, R.C. Haddon. Carbon 72, 82 (2014).

[12] H. Wang, T. Maiyalagan, X. Wang. ACS Catal. 2, 781 (2012).

[13] D. Wei, Y. Liu, Y. Wang, H. Zhang, L. Huang, G. Yu. Nano Lett. 9, 1752 (2009).

[14] L.S. Panchakarla, K.S. Subrahmanyam, S.K. Saha, A. Govindaraj, H.R. Krishnamurthy, U.V. Waghmare, C.N.R. Rao. Adv. Mater. 21, 4726 (2009).

[15] M. Borghei, I. Azcune, P. M Carrasco, J. Sainio, E. Kauppinen, V. Ruiz. Int. J. Hydrogen Energy 39, 12749 (2014).

[16] Y. Fujimoto, S. Saito. Phys. Rev. B 84, 245446 (2011).

[17] M. Pizzochero, O. Leenaerts, B. Partoens, R. Martinazzo, F.M. Peeters. J. Phys. Condens. Matter 27, 425502 (2015).

[18] Y. Wang, Y. Shen, S. Zhu. Catal. Commun. 94, 29 (2017).

[19] Y. Tian, Y. Ye, X. Wang, S. Peng, Z. Wei, X. Zhang, W. Liu. Appl. Catal. A: 529, 127 (2017).

[20] M. Wang, Y. Huang, X. Chen, K. Wang, H. Wu, N. Zhang, H. Fu. J. Alloys Comp. 691, 407 (2017).

[21] K. Gopalakrishnan, K. Moses, A. Govindaraj, C.N.R.Rao. Solid State Commun. 175-176, 43 (2013).

[22] E. Rangel, E. Sansores. Int. J. Hydrogen Energy 39, 6558 (2014).

[23] Z.-Y. Sui, Y.-N. Meng, P.-W. Xiao, Z.-Q. Zhao, Z.-X. Wei, B.H. Han. ACS Appl. Mater. Interfaces 7, 1431 (2015).

[24] S.A. Siadati, E. Vessally, A. Hosseinian, L. Edjlali. Synthetic Met. 220, 606 (2016).

[25] M. Chen, C. Hou, D. Huo, J. Bao, H. Fa, C. Shen. Biosens. Bioelectron. 85, 684 (2016).

[26] L.-H. Pan, S.-H. Kuo, T.-Y. Lin, C.-W. Lin, P.-Y. Fang, H.W. Yang. Biosens. and Bioelectron. 89, 598 (2017).

[27] V. Tozzini, V. Pellegrini. Phys. Chem. Chem. Phys. 15, 80 (2013).

[28] S. Park, R.S. Ruoff. Nature Nanotechnology 4, 217 (2009).

[29] Y.C. Cheng, T.P. Kaloni, Z.Y. Zhu, U. Schwingenschlögl. Appl. Phys. Lett. 101, 073110 (2012).

[30] V.S. Prudkovskiy, K.P. Katin, M.M. Maslov, P. Puech, R. Yakimova, G. Deligeorgis. Carbon 109, 221 (2016).

[31] F. Ou Yang, B. Huang, Z. Li, J. Xiao, H. Wang, H. Xu. J. Phys. Chem. C 112, 12003 (2008).
[32] H.Y. Mao, S. Laurent, W. Chen, O. Akhavan, M. Imani, A.A. Ashkarran, M. Mahmoudi. Chem. Rev. 113, 3407 (2013).

[33] V. Georgakilas, M. Otyepka, A.B. Bourlinos, V. Chandra, N. Kim, K.C. Kemp, P. Hobza, R. Zboril, K.S. Kim. Chem. Rev. 112, 6156 (2012).

[34] D. Bitounis, H. Ali-Boucetta, B.H. Hong, D.-H. Min, K. Kostarelos. Adv. Mater. 25, 2258 (2013).

[35] N.-F. Chiu, S.-Y. Fan, C.-D. Yang, T.-Y. Huang. Biosens. Bioelectron. 89, 370 (2017).

[36] Y. You, J. Deng, X. Tan, N. Gorjizadeh, M. Yoshimura, S.C. Smith, V. Sahajwalla, R.K. Joshi. Phys. Chem. Chem. Phys. 19, 6051 (2017).

[37] S.-M. Choi, S.-H. Jhi, Y.-W. Son. Phys. Rev. B 81, 081407 (2010).

[38] Р.А.Браже, А.И. Кочаев, Р.М. Мефтахутдинов. ФТТ 59, 334 (2017).

[39] B. Liu, C.D. Reddy, J. Jiang, J.A. Baimova, S.V. Dmitriev. Appl. Phys. Lett. 101, 211909 (2012).

[40] S.V. Dmitriev, J.A. Baimova, A.V. Savin, Y.S. Kivshar. Comp. Mater. Sci. 53, 194 (2012).

[41] D.W. Boukhvalov, Y.-W. Son. Chem. Phys. Chem. 13, 1463 (2012).

[42] J.-H. Liao, Y.-J. Zhao, X.-B. Yang. Int. J. Hydrogen Energy 40, 12063 (2015).

[43] X. Wei, L. Mao, R.A. Soler-Crespo, J.T. Paci, J. Huang, S.T. Nguyen, H.D. Espinosa. Nature Commun. 6, 8029 (2015).

[44] P.L. de Andres, J.A. Vergés. Appl. Phys. Lett. 93, 171915 (2008).

[45] К.П. Катин, М.М. Маслов. Хим. физика 30, 41 (2011).

[46] M.M. Maslov, A.I. Podlivaev, K.P. Katin. Mol. Simulation 42 , 305 (2016).

[47] Л.А. Опенов, А.И. Подливаев. ФТТ 58, 821 (2016).

[48] Л.А. Опенов, А.И. Подливаев. ФТТ 58, 1646 (2016).

[49] Л.А. Опенов, А.И. Подливаев. ФТТ 57, 1450 (2015).

[50] Л.А. Опенов, А.И. Подливаев. ФТП 45, 644 (2011).

[51] А.И. Подливаев, К.П. Катин. Письма в ЖЭТФ 92, 54 (2010).

[52] L.A. Openov, A.I. Podlivaev, M.M. Maslov. Phys. Lett. A 376, 3146 (2012).

[53] K.P. Katin, M.M. Maslov. J. Phys. Chem. Solids 108, 82 (2017).

[54] Н.Н. Дегтяренко, К.П. Катин, М.М. Маслов. ФТТ 56, 1415 (2014).

[55] B. Saha, P.K. Bhattacharyya. Comp. Theor. Chem. 1086, 45 (2016).

[56] K.P. Katin, V.S. Prudkovskiy, M.M. Maslov. Phys. Lett. A 381, 2686 (2017).

[57] S. Casolo, O.M. Løvvik, R. Martinazzo, G.F. Tantardini. J. Chem. Phys. 130, 054704 (2009).

[58] С.Ю. Давыдов. ФТТ 59, 825 (2017).

[59] C. Lee, X. Wei, J.W. Kysar, J. Hone. Science 321, 385 (2008). 